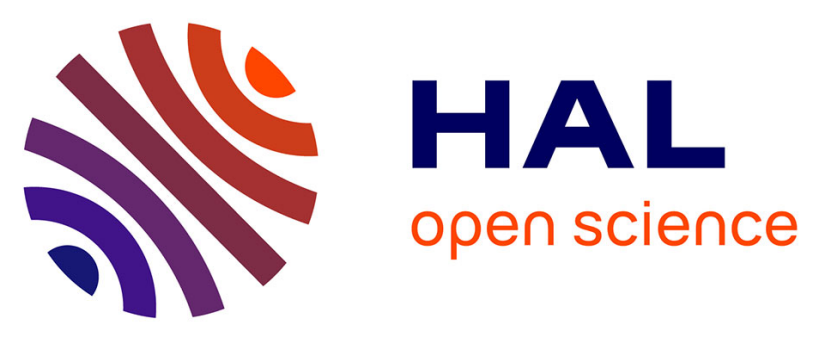

\title{
Elucidation of the Diels-Alder Reaction Kinetics between Diphenylfulvene and Maleimide by Mechanochemistry and in Solution
}

Lori Gonnet, Alain Chamayou, Christiane André-Barrès, Jean-Claude Micheau, Brigitte Guidetti, Tohru Sato, Michel Baron, Michel Baltas, Rachel Calvet

\section{To cite this version:}

Lori Gonnet, Alain Chamayou, Christiane André-Barrès, Jean-Claude Micheau, Brigitte Guidetti, et al.. Elucidation of the Diels-Alder Reaction Kinetics between Diphenylfulvene and Maleimide by Mechanochemistry and in Solution. ACS Sustainable Chemistry \& Engineering, 2021, 9 (12), pp.4453-4462. 10.1021/acssuschemeng.0c08314 . hal-03197711

\section{HAL Id: hal-03197711 https://imt-mines-albi.hal.science/hal-03197711}

Submitted on 15 Apr 2021

HAL is a multi-disciplinary open access archive for the deposit and dissemination of scientific research documents, whether they are published or not. The documents may come from teaching and research institutions in France or abroad, or from public or private research centers.
L'archive ouverte pluridisciplinaire HAL, est destinée au dépôt et à la diffusion de documents scientifiques de niveau recherche, publiés ou non, émanant des établissements d'enseignement et de recherche français ou étrangers, des laboratoires publics ou privés. 


\title{
Elucidation of the Diels-Alder Reaction Kinetics between Diphenylfulvene and Maleimide by Mechanochemistry and in Solution
}

Lori Gonnet,* Alain Chamayou, Christiane André-Barrès, Jean-Claude Micheau, Brigitte Guidetti, Tohru Sato, Michel Baron, Michel Baltas, ${ }^{*}$ and Rachel Calvet*

\begin{abstract}
The Diels-Alder reaction kinetics between diphenylfulvene and maleimide using mechanochemistry and in liquid state is described. This reaction was carried out in solid state using a modified vibratory ball-mill with temperature control and in solution with toluene as solvent. The effect of temperature, ball mass, material, additives, and aging reaction were studied. We reported for the first time the kinetics of a mechanochemical reaction depending on the ratio ball mass/mass of the reagent. A new kinetic model has been established that corresponds well to our experimental data and allows an estimation of the global activation energy. In solution, the retro Diels-Alder reaction was observed. The reaction was second order for the formation of endo
\end{abstract}

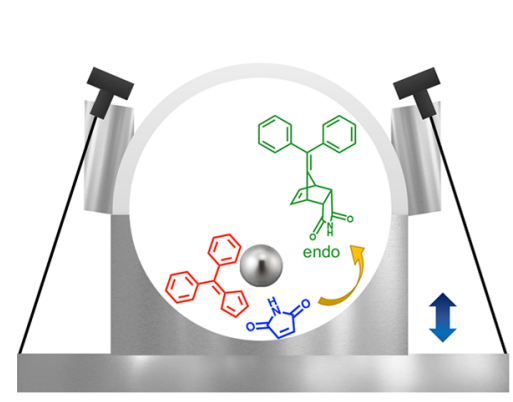

Mechanochemistry

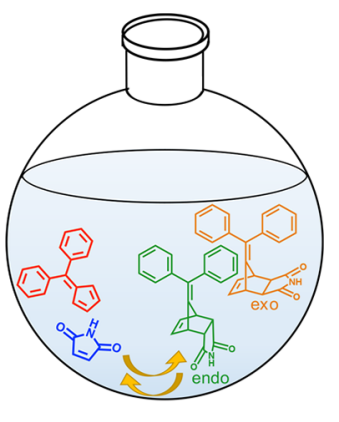

Solution

and exo and first order for the retro Diels-Alder reaction. The grinding method shows many advantages compared to solution: shorter reaction time, total reaction, and better selectivity.

KEYWORDS: Diels-Alder, Mechanochemistry, Ball-milling, Kinetics, Mechanisms

\section{INTRODUCTION}

One of the main goals of organic synthesis is the research and development of efficient and environmentally safe methods. Since the 2000s a lot of stringent regulations for the chemical and pharmaceutical industries have appeared especially in terms of efficiency, waste management, and energy input. All these issues are now addressed and termed as "Green Chemistry", a multifaceted field dealing with what we call the 12 principles of Anastas and Warner. ${ }^{1}$ Among the most important principles are atom economy, preventing the use of solvents, increase of selectivity, minimization of chemical waste and energy. ${ }^{2}$ In relation to the two latter points, in fact organic reactions and processes are usually conducted in solution (mostly organic) and frequently by introducing thermal energy that we have to balance at the end of the reaction with cooling energy as well.

By focusing on the Green Chemistry aspects, the desire to unveil alternative energy sources for chemical transformations under mild conditions led to the discovery of alternative energy systems, ${ }^{3}$ among them the mechanochemical synthesis.

Mechanochemical methods deal with chemical transformations induced by mechanical energy, such as compression, shear, or friction. ${ }^{4-6}$ The sophisticated technique of ballmilling or mechano-milling is the adaptation from the traditional grinding methods using a mortar and pestle.
These mechano-milling methods are generally conducted in vibration mills or planetary mills.

In the past decade, minerals, inorganic compounds, and polymers were the main subjects of investigation. ${ }^{7}$ The organic mechanochemistry has remained undeveloped until the pioneering work reported by Toda in the $1980 \mathrm{~s}^{8}$ and Kaupp. ${ }^{9}$ Due to several advantages, the mechanochemistry area has received significant attention over solution-based chemical methods and process developments. The mechanochemical formation of the carbon-carbon bond (aldol reaction, Michael addition, Morita-Baylis-Hillman, Wittig, Suzuki, cycloaddition reactions), heterocyclic synthesis, carbon-heteroatom formation, multicomponent reactions, and $\mathrm{C}-\mathrm{H}$ functionalization are popularly known in literature. ${ }^{10}$

The mechanistic understanding of the mechanochemical reactions is still unclear. Several models have been proposed, among them: hot spot, magma-plasma, spherical, kinetic, and impulse models are the most well-known. They are supported 
by dislocation and phonon and short-live-active center theories. $^{11-14}$ In-situ analyses by X-ray powder diffraction and Raman spectroscopy have allowed a complete quantification of the kinetics to understand the chemo-mechanical reactivity. ${ }^{15}$ It has also been reported by us and others that some reactions follow zero-order kinetics. ${ }^{16-18}$ In recent years, different phenomenological models have been applied to ball mill reactions to describe the apparent kinetics of mechanochemical transformations. ${ }^{19,20}$ In their principles these approaches take into account the very efficient stirring of the powder in the milling vessel leading to a global chemical homogeneity. However, depending on the critical loading conditions (CLCs), the activation of the transformation occurs in a small fraction of the powder which is trapped in the colliding zone of the milling media. Improving the description of this active trapped volume (geometry, rheological conditions, and so forth) is a way to obtain more precise modeling. A model taking into account the formation of a rubber-like coating around the milling media has been reported to understand the "snowball" kinetic effect observed during a mechanochemical Knoevenagel condensation carried out in ball mill. $^{21}$

The Diels-Alder reaction involves a conjugated diene and an alkene, also called dienophile. This reaction gives rise to a variety of cyclohexene compounds. Several groups have studied the Diels-Alder reaction by mechanochemistry and in particular the mechanochemical parameters such as the frequency of the grinding apparatus, ${ }^{22-24}$ the nature of the bowls and balls material, ${ }^{23,25}$ the number of balls, ${ }^{25}$ the ball mass, and ball diameter. ${ }^{25}$ Finally, we have to mention the studies on the reactivity of retro Diels-Alder reaction by mechanical activation depending on the geometry of the molecules. ${ }^{26,27}$ Except in rare cases, ${ }^{28}$ all these studies on organic mechanochemistry suffer from poor knowledge of the action of mechanochemical forces on chemical reactants.

In order to elaborate and develop future selection rules for mechanochemical reactions, it is essential to point out the increasing need for a more interdisciplinary approach between experimental, physicochemical, and theoretical chemists. ${ }^{29}$ In that respect, we wish to report here a detailed model study of a Diels-Alder reaction between diphenylfulvene and maleimide by a mechanochemical method and in solution. The interest for this reaction was warranted by its kinetics. Diels-Alder reaction is a concerted reaction in which bond breaking in reactants and bond making in products occur in a single step involving a transition state A (Figure 1). The possibility of a retro Diels-Alder pathways is also present. The mechanism of a single step reaction appeared easier to study than that of multi-step reactions.

The choice of the diphenylfulvene 1 was oriented essentially because of its melting point $\left(\mathrm{mp} 81{ }^{\circ} \mathrm{C}\right)$ that permits us to examine the mechanochemical reaction in a suitable range of temperatures where the reactant can remain solid. In this work, we have examined the following mechanochemical parameters, namely: material and the mass of the ball, temperature, influence of grinding auxiliaries, and/or autocatalysis effect and aging. A mechanochemical kinetic model was proposed. The reaction was conducted in toluene and its kinetics was recorded for mechanistic purposes. A comparison between the reaction in solid state and in solution, at the experimental and mechanistic level, is presented.

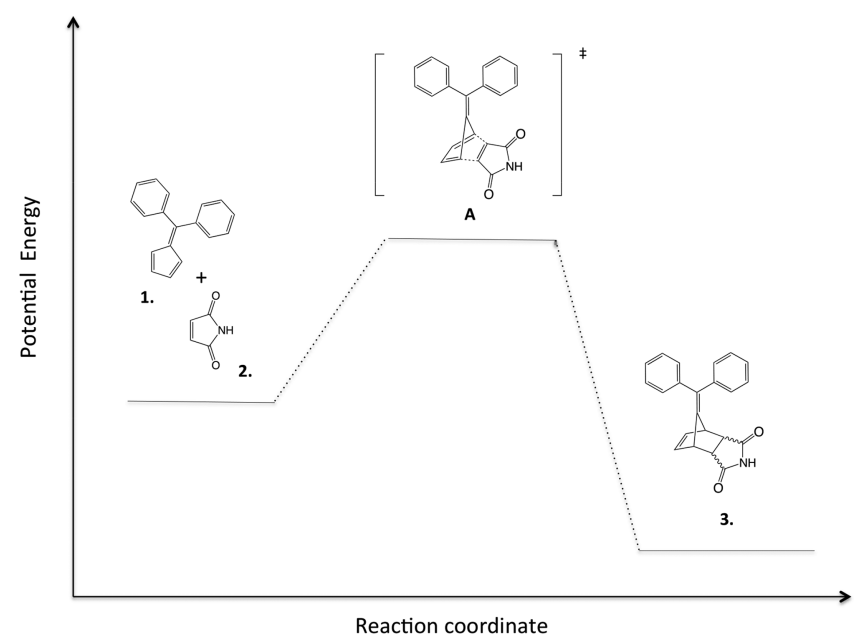

Figure 1. Diels-Alder reaction between diphenylfulvene $\mathbf{1}$ and maleimide 2.

\section{RESULTS AND DISCUSSION}

Mechanochemical Diels-Alder Reaction. The reactions were carried out in a modified vibratory ball-mill Pulverisette 0 (Fritsch, Germany) equipped with a single ball (Figure S1, Supporting Information, SI). A heating/cooling coil was added to the experimental apparatus around the vessel for the temperature setting. The total powder mass of $2.843 \mathrm{~g}$, i.e., $2 \mathrm{~g}$ of diphenylfulvene ( 1 equiv, $8.68 \mathrm{mmol}$ ) and $843 \mathrm{mg}$ of maleimide ( 1 equiv, $8.68 \mathrm{mmol}$ ) was maintained for all experiments. For kinetic sampling, the grinding is stopped after preset grinding periods. Each sample which represents less than $1 \%$ of the total amount of powder in the mill is discarded after analysis.

The reaction products were monitored by analytical HPLC and compounds were extensively examined by NMR spectroscopy (Figure 2). The endo $\mathbf{3 a}$ and exo $\mathbf{3} \mathbf{b}$ products were quantified from their two-proton integration multiplets around 3.7 and $3.5 \mathrm{ppm}$, respectively (see SI Figure S2, for more details).

Influence of Ball Material and Mass. Three types of balls material have been used: brass, copper, and stainless steel. The ball material can influence the kinetics. Higher density balls have been used to obtain higher impact energy. ${ }^{24,25}$ For metalcatalyzed organic reactions, a catalytic effect on the surface of the metals is also possible. ${ }^{30}$ The characteristics of the balls used for the Figure 3 experiments are displayed in Table 1 .

Figure 3 shows that the ball material has no influence on this reaction. On the contrary, a significant effect is observed by changing the ball mass. The reaction proceeds faster when using a heavier ball. Results using a $507 \mathrm{~g}$ stainless steel ball in comparison to a 171 and 109 g, respectively, show clearly significant conversion and an increase of the reaction rate. This is in agreement with the fact that grinding can be faster and more effective with a heavier ball. ${ }^{1,31}$ It is important to note that the endo $\mathbf{3 a}$ /exo $\mathbf{3 b}$ ratio $(98 / 2)$ does not change whatever the operating conditions are.

Influence of Pregrinding of Starting Materials. Diphenylfulvene 1 and maleimide 2 were ground separately during $1 \mathrm{~h}$ at $24{ }^{\circ} \mathrm{C}$. Then, they were mixed together, and the Diels-Alder reaction was conducted at the same temperature, using the same experimental protocol and analytical methods (Figure 4).

Comparison of the two graphs shows a significant acceleration when using the preground starting compounds. 


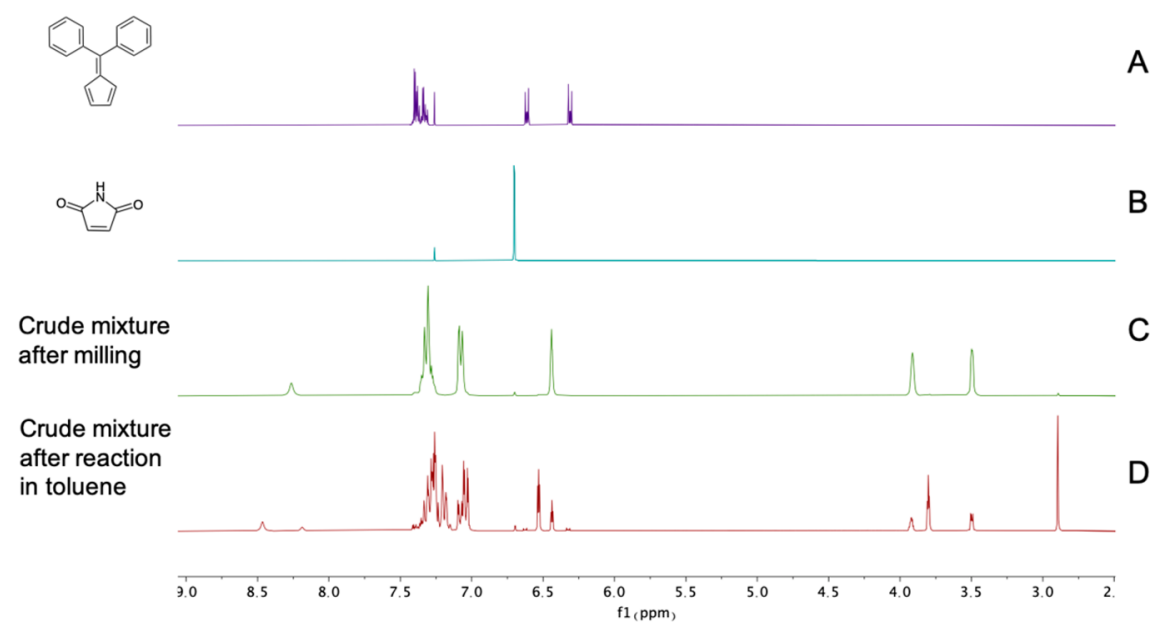

Figure 2. NMR spectra of (A) diphenylfulvene 1, (B) maleimide 2, (C) crude mixture after $8 \mathrm{~h}$ of milling at $24^{\circ} \mathrm{C}$ showing the formation of endo 3a product, and (D) crude mixture after 1 week in toluene at $105{ }^{\circ} \mathrm{C}$ showing the formation of exo $3 \mathbf{b}$ and endo $3 \mathbf{a}$ product.
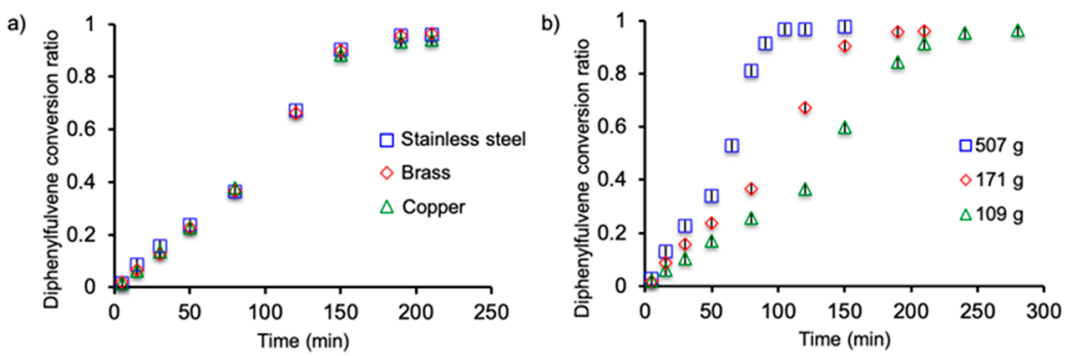

Figure 3. Effect of the ball (a) material and (b) mass (Table 1) on the mechanochemical diphenylfulvene 1 /maleimide 2 Diels-Alder reaction at $44^{\circ} \mathrm{C}$.

Table 1. Milling Balls Used for the Diels-Alder Reaction

\begin{tabular}{clc}
\hline milling ball & ball material & ball mass $(\mathrm{g})^{a}$ \\
\hline BR & brass & 171 \\
CU & copper & 171 \\
SS1 & stainless steel & 171 \\
SS2 & stainless steel & 507 \\
SS3 & stainless steel & 109 \\
\hline
\end{tabular}

${ }^{a}$ All balls have the same diameter $(50 \mathrm{~mm})$ and are empty. They are filled with different solids (sand and lead beads) to change their mass.

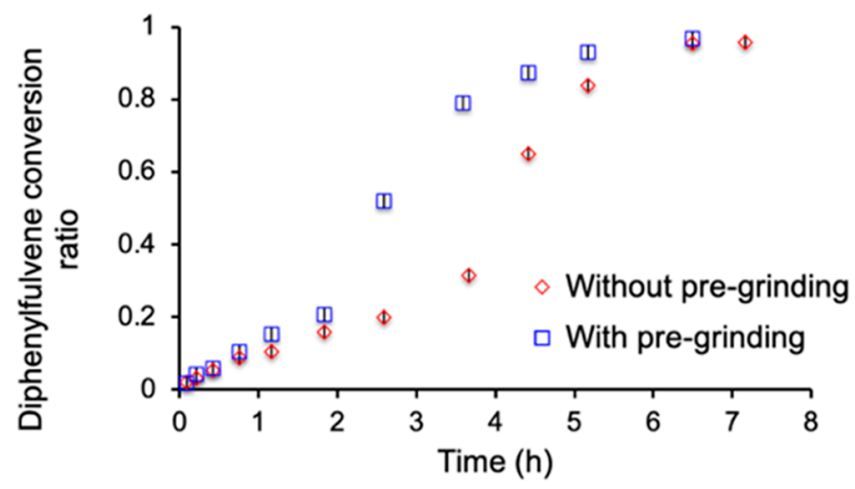

Figure 4. Effect of a pregrinding of starting materials on the mechanochemical diphenylfulvene $1 /$ maleimide 2 Diels-Alder reaction at $24{ }^{\circ} \mathrm{C}$.

After $3.6 \mathrm{~h}$ of grinding, the conversion ratio has reached $80 \%$ vs only $30 \%$ if not preground. In both cases, the final part of the two graphs $(6-8 \mathrm{~h})$ is similar. The conversion is complete and the endo $\mathbf{3 a}$ /exo $\mathbf{3 b}$ ratio $(98 / 2)$ remains the same.

Granulometric analyses were carried-out to estimate the effect of grinding on the particle size. As shown in Figure 5, there is no effect on diphenylfulvene 1 , while the particle size distribution of maleimide 2 becomes bimodal. Smaller particle size is observed $(<50 \mu \mathrm{m})$ but also larger $(1000 \mu \mathrm{m})$, indicating a potential agglomeration of the smaller particles.

The smaller particles size of the maleimide 2 could lead to an increase of the developed surface. Indeed, the higher contact area could induce an acceleration of the reaction rate.

Influence of the Temperature. The reaction between maleimide and diphenylfulvene was also studied under different milling temperatures, varying from $14{ }^{\circ} \mathrm{C}$ to $62{ }^{\circ} \mathrm{C}$ (Figure 6). Results show a significant increase of the reaction rate with the temperature. Indeed, while a total reaction was observed after $6.5 \mathrm{~h}$ of milling at $24{ }^{\circ} \mathrm{C}$, but only $18 \mathrm{~min}$ were needed at $62{ }^{\circ} \mathrm{C}$ to achieve completion. In all experiments, the ratio of endo $3 \mathbf{a}$ /exo $3 \mathbf{b}(98 / 2)$ of the Diels-Alder adduct 3 remained the same in all aliquots examined by analytical HPLC through the whole temperature and time range showing that the high selectivity of the Diels-Alder mechanochemical reaction between diphenylfulvene $\mathbf{1}$ and maleimide $\mathbf{2}$ was not so sensitive in the explored temperature range.

For all the experiments shown above, the evolution of the Diels-Alder mechanochemical reaction follows a sigmoidal curve. An induction period (up to $25 \%$ conversion) is followed in the second part by an acceleration before the rate decreases again as the reaction goes to completion. ${ }^{32}$ This sigmoidal kinetic curve is apparently analogous to those encountered in 
a)

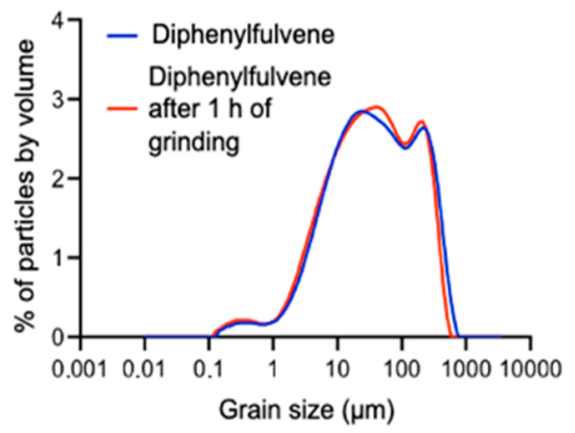

b)

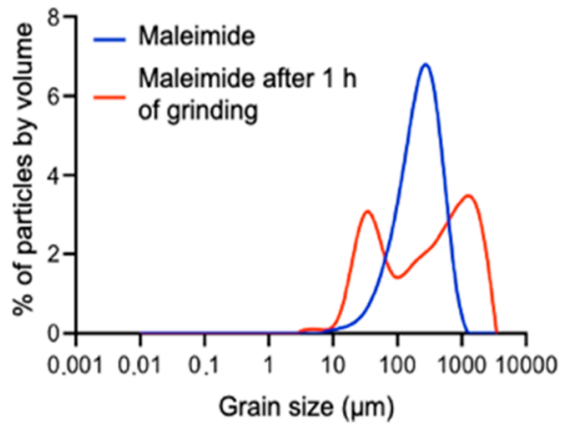

Figure 5. Particle size distribution of (a) diphenylfulvene 1 and (b) maleimide 2 before and after $1 \mathrm{~h}$ of grinding.

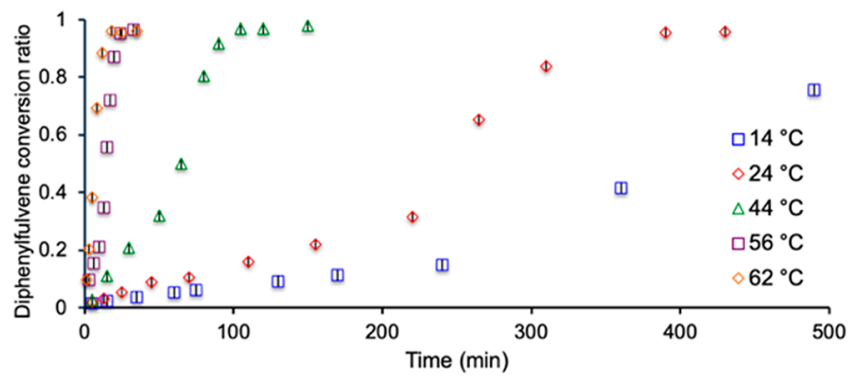

Figure 6. Effect of the temperature on the mechanochemical diphenylfulvene 1 /maleimide 2 Diels-Alder reaction.

autocatalysis. $^{33}$ This is a strong indication of the presence of some feedback process whose detailed nature remains to be understood. In that respect, we wished to examine if an autocatalysis-like phenomenon issued by the final products might be responsible of the acceleration process. A reaction was thus performed at $24{ }^{\circ} \mathrm{C}$ in the presence of $20 \%$ of the Diels-Alder adduct 3 corresponding to the amount present after $2 \mathrm{~h}$ of grinding during previous experiments.

The results obtained show that the addition of $20 \%$ of final product 3 increases the reaction rate and that the sigmoidal shape of the kinetics is lost (no more an acceleration period). A similar result with an even better response has been found in a test experiment conducted by introducing $20 \%$ of pyrogenic silica instead of the Diels-Alder adduct 3, always keeping the same total mass (Figure 7). Pyrogenic silica was selected because it has a median diameter $d_{50}$ close to the final product (Table 2) and because it is well-known that silica acts as a catalyst for the Diels-Alder reaction. ${ }^{34}$ In this way, we could

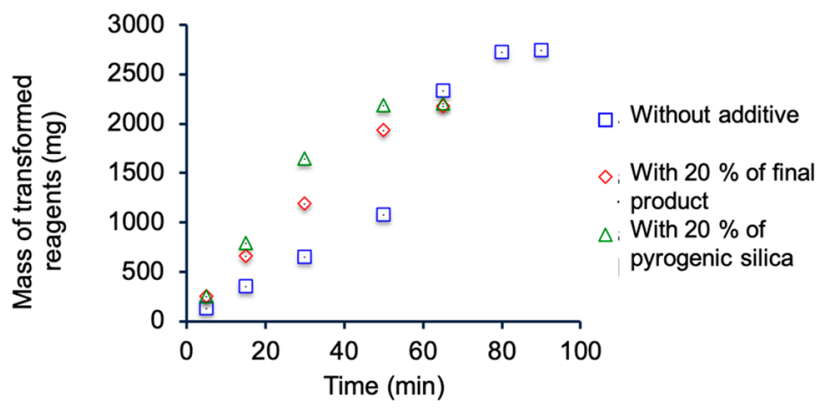

Figure 7. Effect of various additives (final product and grinding auxiliary) on the mechanochemical diphenylfulvene 1 /maleimide 2 Diels-Alder reaction. All the experiments were carried out with the same total mass of powder $(2.843 \mathrm{~g})$.
Table 2. Comparison of the Grain Sizes of the Various Additives According to Their $d_{50}$ Median Diameter

\begin{tabular}{lc}
\multicolumn{1}{c}{ material } & $d_{50}(\mu \mathrm{m})^{a}$ \\
final product 3 & 32 \\
sand & 246 \\
silica gel & 125 \\
pyrogenic silica & 37
\end{tabular}

${ }^{a} d_{50}:$ median diameter

be able to know if the final product 3 acts as a catalyst, like silica.

From these experiments, it can be inferred that under mechanochemical conditions a positive feedback mechanism is operating. Such a situation is often encountered in the framework of autocatalyzed reactions. ${ }^{35}$ However, in this specific case, such autocatalysis-like evolution is not due to a simple molecular mechanism, but more likely to a complex combination of rheological and physicochemical phenomena. From this point of view, the silica acceleration effect is instructive, because it was assumed to originate from a preassociation mechanism resulting from a rigid anchoring of the reaction partners within a critical bonding distance. Under ball-milling, such preassociation could occur in tiny cocrystal phases whose influence or size is slowly increasing as the reaction processes. Such an assumption is supported by the aging studies where it is shown that the rate increases after previous cogrinding, but above all, that the sigmoidal shape remains if the cogrinding is short, but disappears after longer cogrinding. Traces or seeds of such tiny cocrystal phases are likely to persist within the added Diels-Alder adduct 3, hence the acceleration effect.

Aging. During all the grinding experiments detailed above, it was observed that the Diels-Alder reaction continues even if the grinding stops (aging). Indeed, some chemical reactions can occur spontaneously between solid reagents, in some cases facilitated by humidity, organic vapors, the addition of catalysts, heating, or brief grinding. ${ }^{36}$ In our case, this phenomenon does not take place when diphenylfulvene and maleimide are simply mixed without previous cogrinding. So, the aging reaction was studied after $15 \mathrm{~min}$ and $1 \mathrm{~h}$ of grinding at $24{ }^{\circ} \mathrm{C}$ (Figure 8 ). The conversion rates are $4 \%$ and $8 \%$, respectively, after $15 \mathrm{~min}$ and $1 \mathrm{~h}$ of grinding. The reaction mixture was then left at room temperature $\left(22{ }^{\circ} \mathrm{C}\right)$ and its evolution was followed by analytical HPLC. The conversion rate is significantly faster after a longer grinding time. This may be due to the larger creation of a cocrystal after $1 \mathrm{~h}$, in which the molecules are positioned to react. This was also confirmed 


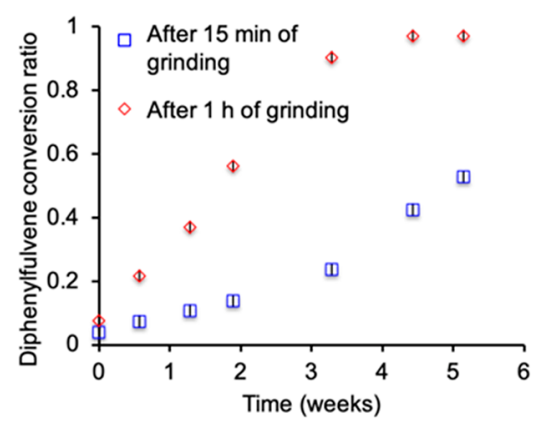

Figure 8. Effect of substrate aging (pregrinding) on the mechanochemical diphenylfulvene 1 /maleimide 2 Diels-Alder reaction.

by calorimetric analyses, where a higher heat release was observed after longer grinding times (SI Figure S4).

Kinetics. The reaction kinetics of Diels-Alder by mechanochemistry was then studied to have a better understanding of the mechanism of the transformation during grinding. Experiments concerning the influence of the ball mass and the ratio ball mass/reagent mass lead to the hypothesis that the specific energy brought to the reagents seems to be one of the major parameters of the apparent kinetics of the transformation. In that respect, a preliminary semiempirical model can be proposed and its adequacy will be verified with the set of experimental results. During a reaction by grinding, the ratio ball mass/mass of the reagent influences the rate of the transformation notably through the creation of contact surface around the active grains under the effect of the grinding. The variation of the mass $(m)$ of a reagent can be considered to be dependent on the ratio of the ball mass $\left(M_{\mathrm{b}}\right)$ to $m$ (eq 1). This equation has therefore been integrated (eq 2 ) and reformulated (eq 3 ) according to the conversion rate $X$ (eq 4).

$$
-\frac{d m}{d t}=k\left(\frac{M_{\mathrm{b}}}{m}\right)
$$

The integration of eq 1 leads to eq 2:

$$
\frac{1}{2}\left(m_{0}^{2}-m^{2}\right)=k M_{\mathrm{b}} t
$$

The mass of reagent can be replaced by its expression with the conversion rate $X$ and eq 2 becomes eqs 3 and 4

$$
2 X-X^{2}=2 k \frac{M_{\mathrm{b}}}{m_{0}^{2}} t
$$

a)

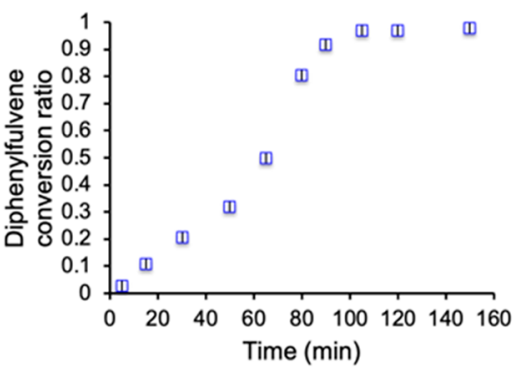

$$
2 X-X^{2}=A_{t} \text { with } A=2 k \frac{M_{\mathrm{b}}}{m_{0}^{2}}
$$

with

$$
X=\frac{m_{0}-m}{m_{0}}
$$

This simple kinetic model was then applied to our reaction records performed with our mechanochemical apparatus. For all experiments, a linearization of the experimental points was obtained as much as more than $80 \%$ of the reaction (Figure 9). This result suggests that this model can be used as a first approximation for the Diels-Alder reaction by mechanochemistry. However, as this model is limited to the early part of the reaction, the slowed-down final part of the curve is not expected to be reproduced. Let us say that such a slow-down of the final part is common to all kinds of kinetics and that its mechanistic information is relatively poor.

The apparent rate constants $A$ delivered by this kinetic model are gathered in Table 3.

Table 3. Apparent Rate Constants at Different Temperatures

$\begin{array}{ccrcc}\text { entry } & \text { grinding temperature }\left({ }^{\circ} \mathrm{C}\right) & \text { time }(\mathrm{min}) & \mathrm{A}\left(\mathrm{min}^{-1}\right) & \mathrm{R}^{2 a} \\ \mathbf{1} & 14 & 5 \text { to } 250 & 1.03 \times 10^{-3} & 0.993 \\ \mathbf{2} & 24 & 5 \text { to } 220 & 2.24 \times 10^{-3} & 0.995 \\ \mathbf{4} & 44 & 5 \text { to } 80 & 1.16 \times 10^{-2} & 0.992 \\ \mathbf{5} & 56 & 1.5 \text { to } 13 & 3.61 \times 10^{-2} & 0.966 \\ \mathbf{6} & 62 & 1.5 \text { to } 8 & 1.11 \times 10^{-1} & 0.996\end{array}$

${ }^{a}$ Note the measurement time interval and the good correlation coefficients confirming the linearization by the kinetic model.

The relationship between temperature and reaction rate constant is generally described by the Arrhenius equation (eq 6) and its linearized form (eq 7). The Arrhenius equation can be also valid to determine the activation energy of solid state reactions. $^{37,38}$

$$
\begin{aligned}
& k=k_{0} e^{-E_{\mathrm{a}} / R T} \\
& \ln k=\ln k_{0}-\frac{E_{\mathrm{a}}}{R} \frac{1}{T}
\end{aligned}
$$

where $k$ the rate constant, $k_{0}$ the pre-exponential factor, $E_{\mathrm{a}}$ the activation energy for the reaction, $R$ the universal gas constant, and $T$ the absolute temperature.

According to eq 6, the plot of $\log A$ vs $1 / T$ is shown on Figure 10. All points from 14 to $56{ }^{\circ} \mathrm{C}$ are nicely aligned.

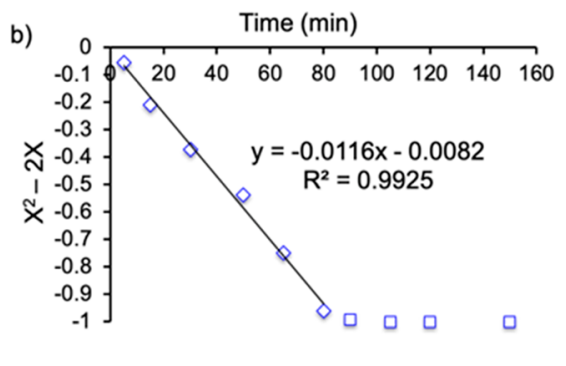

Figure 9. (a) Evolution of the diphenylfulvene conversion rate at $44{ }^{\circ} \mathrm{C}$ and (b) linearization by the kinetic model (eq 3a,b). The points are aligned up to approximately $X=0.88$ (i.e., when $X^{2}-2 X=-0.99$ ). 


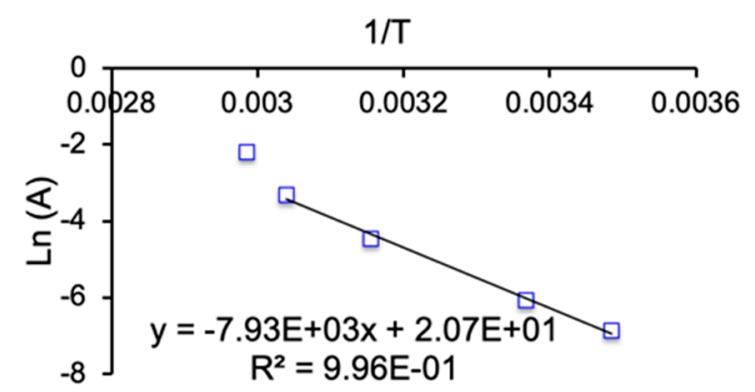

Figure 10. Determination of the activation energy of the mechanochemical diphenylfulvene 1/maleimide 2 Diels-Alder reaction.

However, the last point at $62{ }^{\circ} \mathrm{C}$ is a slight departure. This deviation from linearity is significant since all experiments were made in triplicate. One possible explanation could be related to the endothermic effect of the melting of the powder mixture. DSC measurements were performed to find out if a possible contact eutectic melting formation was occurring (Figure 11).

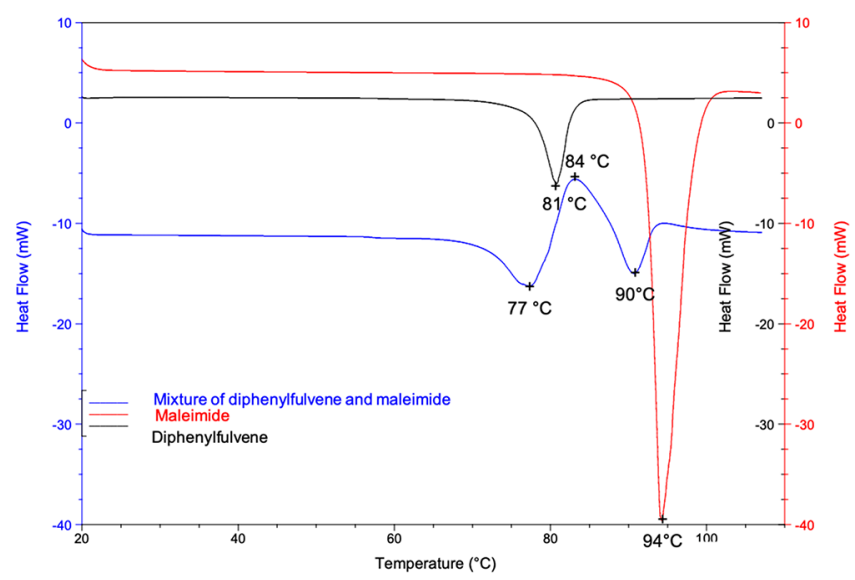

Figure 11. DSC thermograms of diphenylfulvene 1, maleimide 2 , and mixture of reagents

a)

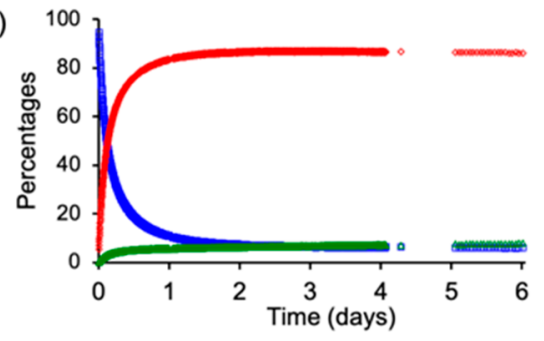

For diphenylfulvene $\mathbf{1}$ and maleimide $\mathbf{2}$, an endothermic peak, corresponding to the melting points, was found $\left(81{ }^{\circ} \mathrm{C}\right.$ for diphenylfulvene 1 and $94{ }^{\circ} \mathrm{C}$ for maleimide 2 ). Then the equimolar mixture of the reagents was analyzed. We can observe the beginning of the melting endotherm of diphenylfulvene 1 at $77^{\circ} \mathrm{C}$ followed by an exotherm at 84 ${ }^{\circ} \mathrm{C}$ and melting endotherm of maleimide 2 at $90{ }^{\circ} \mathrm{C}$. A slight shift for both melting endothermic peaks is observed because the masses of each of the reagents, diphenylfulvene $\mathbf{1}$ and maleimide 2, present in the mixture are smaller than those of the same reagents analyzed alone (for each analysis, a total sample mass of $5 \mathrm{mg}$ is analyzed). In addition, the endotherm of diphenylfulvene $\mathbf{1}$ is not fully visible due to the exothermic peak present at $84^{\circ} \mathrm{C}$. This exothermic peak can be attributed to the start of the reaction and occurs during the fusion of diphenylfulvene $\mathbf{1}$, i.e., at the moment when the reagents come into contact. In the case of the grinding experiment at $62{ }^{\circ} \mathrm{C}$, the melting temperature of diphenylfulvene $\left(77-81^{\circ} \mathrm{C}\right)$ may be reached locally at the impact point. The melting endotherm is probably confounding the temperature measurements by extracting heat from the system. At lower temperatures (from 14 to $56{ }^{\circ} \mathrm{C}$ ), the reaction between the solids prevails, as indicated by the straightforward linear decrease of $\ln A$ with $1 /$ $T$.

An activation energy of $67 \mathrm{~kJ} / \mathrm{mol}$ was estimated for the linear range, i.e., up to $56{ }^{\circ} \mathrm{C}$. This value is of the same order of magnitude as that reported in the literature for other mechanochemical Diels-Alder reactions (between 30 and $115 \mathrm{~kJ} / \mathrm{mol}) .^{39-41}$ Previous work on the synthesis of 2,3diphenylquinoxaline using our mechanochemical apparatus showed an activation energy between 15 and $92 \mathrm{~kJ} / \mathrm{mol}^{17}$ Quantum calculations are in progress to determine the best transition state geometries. ${ }^{42}$ They will be the subject of a further publication.

Liquid State Diels-Alder Reaction. For comparison purposes, the reaction between diphenylfulvene $\mathbf{1}$ and maleimide 2 was also carried out in toluene at different temperatures: 70,80 , and $105{ }^{\circ} \mathrm{C}$ and monitored by NMR (Figure 12a,b,d) and HPLC (Figure 12c).

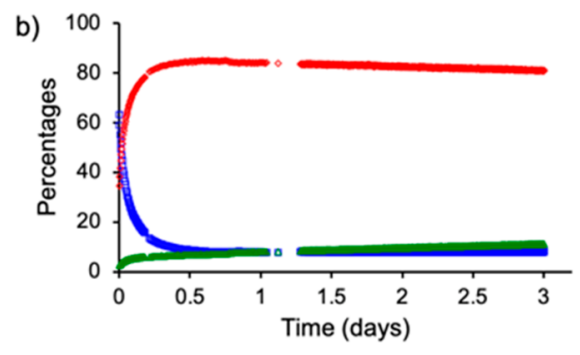

$\square$ Diphenylfulvene $\diamond$ Endo product $\triangle$ Exo product
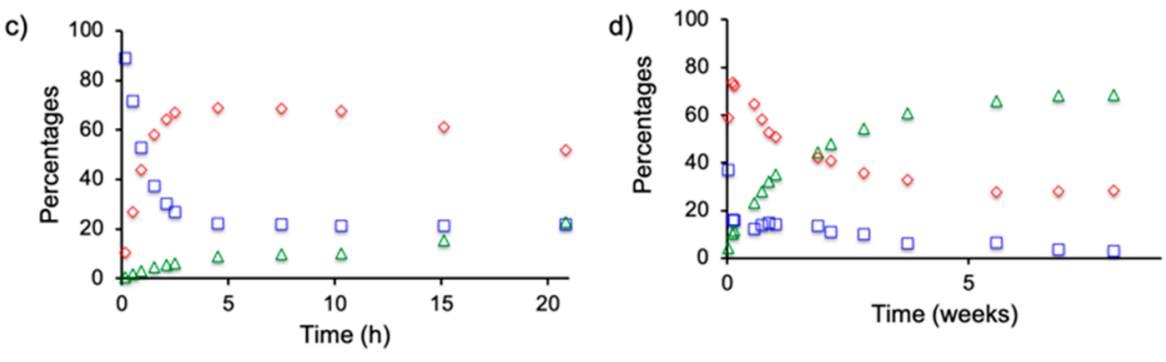

Figure 12. Evolution of diphenylfulvene 1 /maleimide 2 Diels-Alder reaction in toluene at different temperatures: (a) $70{ }^{\circ} \mathrm{C}$, (b) $80{ }^{\circ} \mathrm{C}$ with NMR monitoring, (c) $105{ }^{\circ} \mathrm{C}$ with HPLC monitoring, and (d) $105{ }^{\circ} \mathrm{C}$ with NMR monitoring. Note the longer time scale in d. 
At $70{ }^{\circ} \mathrm{C}$ (Figure 12a) a sharp decrease of the diphenylfulvene $\mathbf{1}$ in less than a day and a stabilization at around $10 \%$ is witnessed. At $80{ }^{\circ} \mathrm{C}$ (Figure 12b), this consumption is faster, the stabilization at $10 \%$ is reached in about $10 \mathrm{~h}$ of reaction. Simultaneously, it is noteworthy to consider the evolution of the endo $3 \mathbf{a}$ /exo $\mathbf{3 b}$ ratio moving slightly from $93 / 7$ to $91 / 9$ after 6 days at $70{ }^{\circ} \mathrm{C}$ and more significantly from $92 / 8$ to $88 / 12$ after 3 days at $80{ }^{\circ} \mathrm{C}$. All these variations suggest the presence of a retro-Diels reaction. A very interesting result is also observed when conducting the reaction at $105^{\circ} \mathrm{C}$ (Figure $12 \mathrm{c}, \mathrm{d}$ ). The NMR monitoring for a more than a 5-week period (Figure 12d) and the HPLC one for more than $20 \mathrm{~h}$ (Figure 12c) show a sharp decrease of the starting diphenylfulvene 1 during the first hours of the reaction and then a slight increase before a further decrease (5\% present after 5 weeks). At the same time, while the endo adduct $3 a$ is the major one in the beginning of the reaction, it decreases and becomes the minor one after 15 days. At $105{ }^{\circ} \mathrm{C}$, the endo $3 \mathrm{a} /$ exo 3 b ratio evolves from $88 / 12$ at $20 \mathrm{~h}$ to $29 / 71$ after 8 weeks.

These results clearly show that (i) the endo product $3 a$ is the kinetically controlled adduct, (ii) the reaction is not complete even at the higher temperature after several weeks, and (iii) the retro Diels-Alder reaction is also operating. The following mechanism is assumed for the diphenylfulvene 1/ maleimide 2 Diels-Alder reaction in solution (Figure 13).

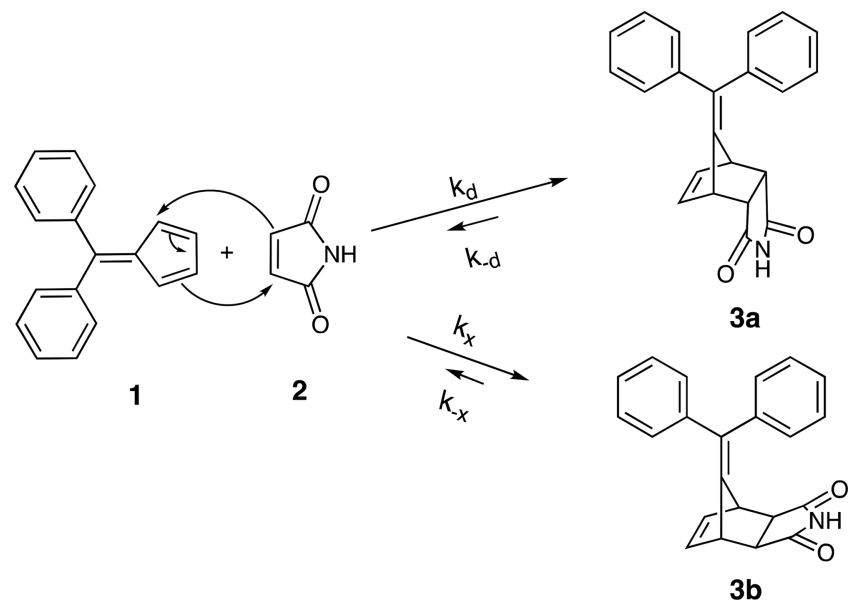

Figure 13. Proposed mechanism for the diphenylfulvene $1 /$ maleimide 2 Diels-Alder reaction in liquid state.

We assume that the formation of the endo $3 \mathbf{a}$ and exo $\mathbf{3 b}$ products is second order (eq 8) and that the retro Diels-Alder is first order (eqs 9 and 10). The differential equations in agreement with this model are as follows:

$$
\begin{aligned}
& \frac{d[\mathrm{diph}]}{d t}=\frac{d[\mathrm{mal}]}{d t}=-\left(k_{d}+k_{x}\right)[\mathrm{diph}][\mathrm{mal}]+k_{-d}[\text { endo }]+k_{-x}[\text { exo }] \\
& \frac{d[\text { endo }]}{d t}=k_{d}[\mathrm{diph}][\mathrm{mal}]-k_{-d}[\text { endo }] \\
& \frac{d[\text { exo }]}{d t}=k_{x}[\mathrm{diph}][\mathrm{mal}]-k_{-x}[\text { exo }]
\end{aligned}
$$

These differential equations can be numerically integrated with any empirical values of the rate constants. To obtain the experimental rate constants, a homemade iterative optimization software was used. ${ }^{43}$ This software allows us to simulate the evolution of the substrate and the products and to adjust the rate constants until the simulated curves exactly fit the experimental kinetic data. The results of the optimization of the kinetics for the reactions at $70{ }^{\circ} \mathrm{C}, 80{ }^{\circ} \mathrm{C}$, and $105{ }^{\circ} \mathrm{C}$ are shown in Figure 14. The experimental points overlap perfectly for the reactions at $70{ }^{\circ} \mathrm{C}$ and $80{ }^{\circ} \mathrm{C}$ (Figure 14a,b) with the simulated curves. For the experiments at $105{ }^{\circ} \mathrm{C}$, the curves (kinetic model) are very close to the markers but do not fully overlap. Indeed, for the reactions at $70{ }^{\circ} \mathrm{C}$ and $80{ }^{\circ} \mathrm{C}$, the reaction monitoring was carried out directly in the NMR tube inside the NMR spectrometer at the desired temperature, whereas for the reactions at $105{ }^{\circ} \mathrm{C}$, the conversion rates were determined for the 8 weeks period by HPLC (Figure 14c) and by NMR analyses but after sampling in a flask. Experiments at $105{ }^{\circ} \mathrm{C}$ may thus be subject to more experimental error. The rate constants obtained are listed in Table 4.

These results show that the endo product $3 a$ is the kinetic one consequently, the exo product $\mathbf{3 b}$ is the thermodynamic one. The equilibration is very slow since it occurs through the retro Diels-Alder reaction. The rate constant $k_{-x}$ is very small and can only be determined precisely from the long-term experiment at $105{ }^{\circ} \mathrm{C}$. However, the reaction at $105{ }^{\circ} \mathrm{C}$ for 8 weeks is too long to allow the relatively fast $k_{d}$ and $k_{-d}$ to be accurately obtained.

Figure 15 shows the application of the Arrhenius equation to the rate constants determined in solution. Table 5 summarizes the activation energies obtained in liquid state and by mechanochemistry.

In mechanochemistry, the apparent rate constants measure the overall rate of this process from the maleimide $\mathbf{2}$ and diphenylfulvene 1 consumption including grinding, endo $3 \mathrm{a} /$ exo $3 \mathbf{b}$ product formation, and potentially retro-Diels-Alder, thus also resulting in an activation energy $E_{\mathrm{a}}$ value of the overall process. On the contrary, in liquid medium, the rate constants and the $E_{\mathrm{a}}$ have been estimated from the main steps. Therefore, these values cannot be compared directly. In solution, the endo product $3 a$ is the kinetic one with an activation energy determined at $38 \mathrm{~kJ} / \mathrm{mol}$, while the thermodynamically more stable exo product $3 \mathbf{b}$ has an activation energy of $50 \mathrm{~kJ} / \mathrm{mol}$ (Table 5). The $E_{\mathrm{a}}$ of endo product 3a for the retro Diels-Alder reaction is quite high $(121 \mathrm{~kJ} / \mathrm{mol})$. Under mechanochemical conditions detailed before, the Diels-Alder reaction between diphenylfulvene and maleimide is much more efficient (total reaction), faster, and potentially too fast to show any retro Diels-Alder transformations, i.e., no endo/exo equilibration was observed. We think that this lack of mechanochemical equilibration is the consequence the high retro-Diels activation energy $(121 \mathrm{~kJ} /$ $\mathrm{mol}$ ). Such a value cannot be attained when using our mechanochemical apparatus. The pre-exponential factor, $k_{0}$, is higher by mechanochemistry. This result means that molecular state encounters are more efficient with this method, which is consistent with a faster transformation rate by mechanochemistry than in solution [total reaction after $18 \mathrm{~min}$ at $62{ }^{\circ} \mathrm{C}$ using mechanochemistry and reaction equilibrium after 2 days in toluene at $\left.70{ }^{\circ} \mathrm{C}(\mathrm{C}=2.9 \mathrm{~mol} / \mathrm{L})\right]$.

\section{CONCLUSIONS}

We reported here a detailed model study of a Diels-Alder reaction between diphenylfulvene $\mathbf{1}$ and maleimide 2 by a mechanochemical method and in toluene. The effect of the experimental conditions for the mechanochemical reaction were studied (material and mass of the ball, temperature, 

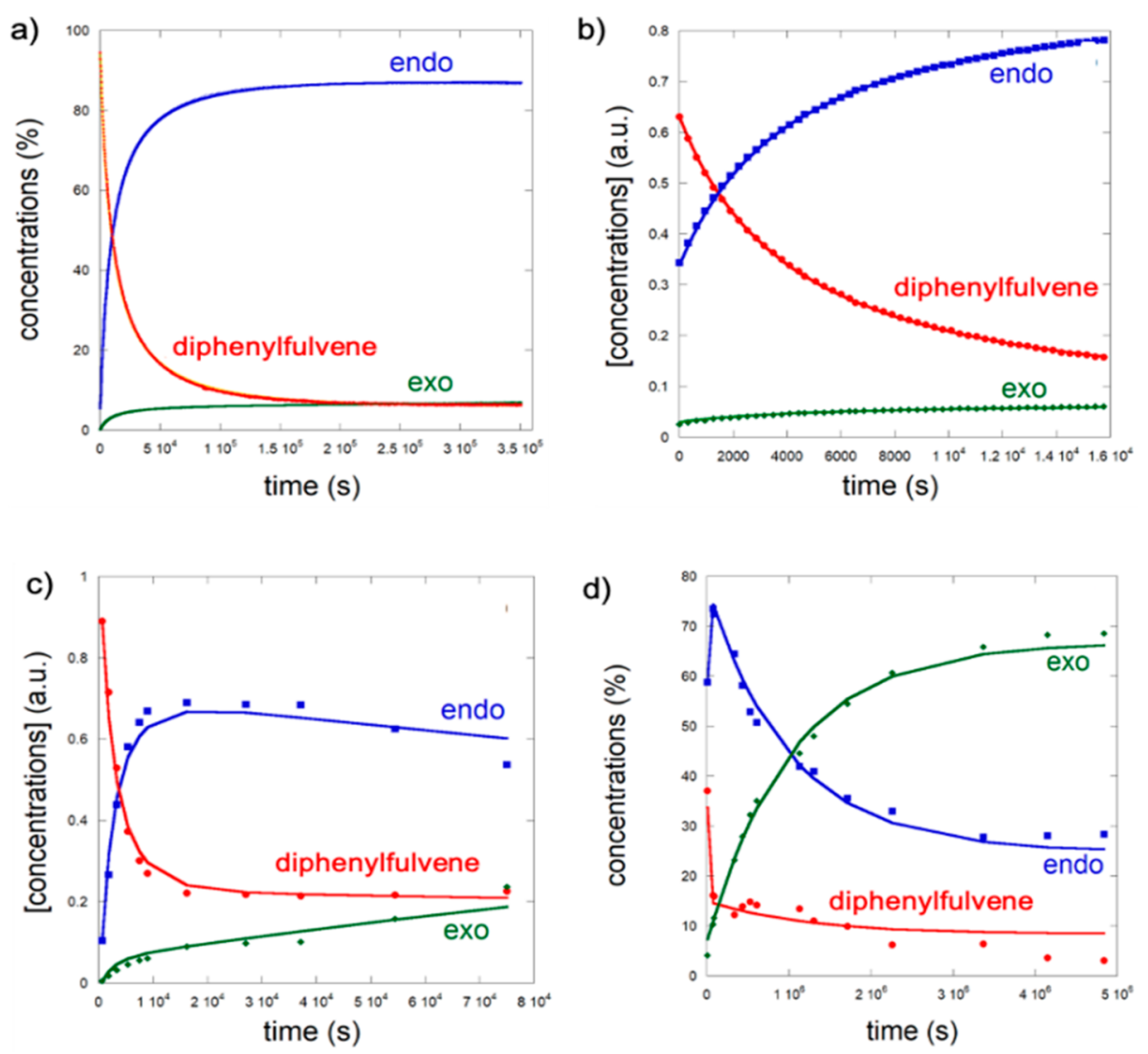

Figure 14. Kinetic modeling of the Diels-Alder reaction (a) evolution at $70{ }^{\circ} \mathrm{C}$ for 4 days; (b) at $80{ }^{\circ} \mathrm{C}$ for 4 h. Both experiments "a" and "b" were monitored by NMR. Note the perfect correspondence between the experimental points and the simulated model (c) at $105^{\circ} \mathrm{C}$ for $2 \mathrm{~h}$ and $(\mathrm{d})$ at $105{ }^{\circ} \mathrm{C}$ for 8 weeks. Experiment "c" was monitored by HPLC and "d" by NMR. Note the relatively good reproduction of the evolutions by the model.

Table 4. Rate Constants from the Kinetic Analysis of the Diphenylfulvene 1/Maleimide 2 Diels-Alder Reaction in Solution ${ }^{a, b}$

$\begin{array}{ccccc}\text { temperature }\left({ }^{\circ} \mathrm{C}\right) & k_{d}\left(\mathrm{M}^{-1} \cdot \mathrm{s}^{-1}\right) & k_{-d}\left(\mathrm{~s}^{-1}\right) & k_{x}\left(\mathrm{M}^{-1} \cdot \mathrm{s}^{-1}\right) & k_{-x}\left(\mathrm{M}^{-1} \cdot \mathrm{s}^{-1}\right) \\ 70 & 3.3 \times 10^{-3} & 4.3 \times 10^{-7} & 2.3 \times 10^{-4} & \sim 0 \\ 80 & 5.3 \times 10^{-3} & 3.0 \times 10^{-6} & 3.2 \times 10^{-4} & \sim 0 \\ 105^{a} & 1.1 \times 10^{-2} & 2.5 \times 10^{-5} & 1.2 \times 10^{-3} & \sim 0 \\ 105^{b} & 0.7 \text { to } 1.2 \times 10^{-1} & 5.6 \text { to } 10 \times 10^{-4} & 9.3 \times 10^{-4} & 2.9 \times 10^{-7}\end{array}$

${ }^{a}$ Short-term reaction monitored by HPLC. ${ }^{b}$ Long-term reaction monitored by NMR.

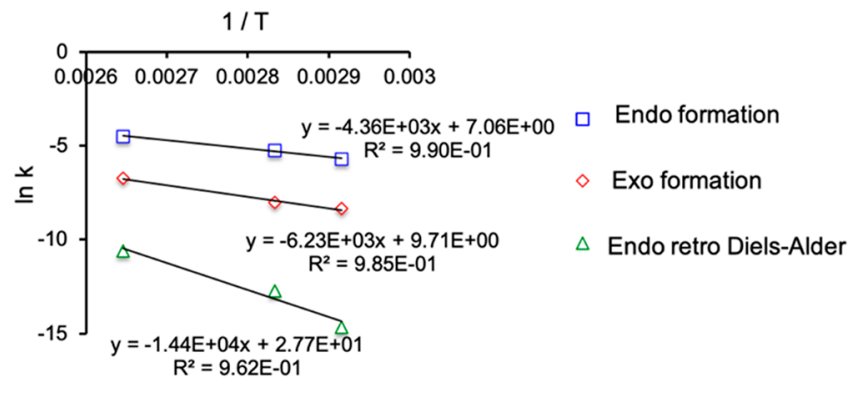

Figure 15. Determination of the activation energies of the liquid state diphenylfulvene 1 /maleimide 2 Diels-Alder direct- and retroreactions.

influence of grinding auxiliaries, and aging). Our results demonstrated the influence of the ball mass, temperature, and an aging phenomenon. Grinding auxiliaries play the same role as the Diels-Alder adducts upon the reaction progress indicating only a positive mechanical role of the adducts/ auxiliaries by accelerating the reaction when present at $20 \%$. In mechanochemistry, the transformation is complete, and the very selective ratio of the Diels-Alder adducts 3 remains unchanged (endo 3a/exo 3b 98/2) through the whole temperature and time range. This is an indication that the Diels-Alder mechanochemical reaction between diphenylfulvene $\mathbf{1}$ and maleimide 2 is very selective. ${ }^{44}$ The deleterious retro Diels-Alder reaction is inhibited. A kinetic model linking the transformation kinetic to the mechanical energy given to the reactants through the ratio mass of the ball/mass of reactants has been proposed. It fits well with our experimental data up to $88 \%$ conversion and allows an estimation of the global activation energy.

In toluene, the reaction is much slower and not complete. The corresponding retro Diels-Alder is occurring. The kinetic endo product $3 \mathbf{a}$ is slowly transformed into the thermodynamic exo $3 \mathbf{b}$. The ratio endo $3 \mathbf{a} /$ exo $3 \mathbf{b}$ evolves during 6 weeks at $105{ }^{\circ} \mathrm{C}$ from $88 / 12$ to $29 / 71$. It has been found that the retro-Diels reaction exhibits a high activation energy (121 $\mathrm{kJ} / \mathrm{mol}$ ). We believe that this high value explains why the retro-Diels reaction does not occur in our mechanochemistry 
Table 5. Activations Energies of the Diphenylfulvene 1/Maleimide 2 Diels-Alder Direct- and Retro-Reactions by Mechanochemistry and in the Liquid State

\begin{tabular}{|c|c|c|c|}
\hline & & $E_{\mathrm{a}}(\mathrm{kJ} / \mathrm{mol})$ & $\ln k_{0}$ \\
\hline mechanochemistry & & 67 & 20 \\
\hline \multirow[t]{3}{*}{ liquid state } & endo product formation & 38 & 7 \\
\hline & exo product formation & 50 & 10 \\
\hline & endo product retro Diels-Alder & 121 & 28 \\
\hline
\end{tabular}

apparatus allowing us to obtain the kinetically controlled adduct with very high selectivity.

\section{ASSOCIATED CONTENT}

\section{SI Supporting Information}

The Supporting Information is available free of charge at https://pubs.acs.org/doi/10.1021/acssuschemeng.0c08314.

Experimental procedures, computation parameters, DSC, calorimetric measurements, and $1 \mathrm{H}$ and $13 \mathrm{C}$ spectral data of all compounds (PDF)

\section{AUTHOR INFORMATION}

\section{Corresponding Authors}

Lori Gonnet - Université de Toulouse, IMT Mines Albi, UMR CNRS 5302, Centre RAPSODEE, Campus Jarlard, Allée des Sciences, Albi cedex 09 F-81013, France; LSPCMIB, Université de Toulouse, CNRS UMR 5068, Université Toulouse III-Paul Sabatier, 31062 Toulouse, France; ○ orcid.org/0000-0001-6866-7387; Email: lori.gonnet@ mines-albi.fr

Michel Baltas - LSPCMIB, Université de Toulouse, CNRS UMR 5068, Université Toulouse III-Paul Sabatier, 31062 Toulouse, France; 이이이.org/0000-0002-8785-7095; Email: michel.baltas@lcc-toulouse.fr

Rachel Calvet - Université de Toulouse, IMT Mines Albi, UMR CNRS 5302, Centre RAPSODEE, Campus Jarlard, Allée des Sciences, Albi cedex 09 F-81013, France; ○ orcid.org/0000-0002-5515-0976; Email: rachel.calvet@ mines-albi.fr

\section{Authors}

Alain Chamayou - Université de Toulouse, IMT Mines Albi, UMR CNRS 5302, Centre RAPSODEE, Campus Jarlard, Allée des Sciences, Albi cedex 09 F-81013, France; (1) orcid.org/0000-0002-2060-1837

Christiane André-Barrès - LSPCMIB, Université de Toulouse, CNRS UMR 5068, Université Toulouse III-Paul Sabatier, 31062 Toulouse, France; 이이.org/0000-00028973-1875

Jean-Claude Micheau - Laboratoire des IMRCP, Université de Toulouse, CNRS UMR 5623, Université Toulouse III-Paul Sabatier, 31062 Toulouse, France; ○ orcid.org/ 0000-0002-1193-4306

Brigitte Guidetti - LSPCMIB, Université de Toulouse, CNRS UMR 5068, Université Toulouse III-Paul Sabatier, 31062 Toulouse, France

Tohru Sato - Fukui Institute for Fundamental Chemistry, Kyoto University, Kyoto 606-8103, Japan

Michel Baron - Université de Toulouse, IMT Mines Albi, UMR CNRS 5302, Centre RAPSODEE, Campus Jarlard, Allée des Sciences, Albi cedex 09 F-81013, France; (1) orcid.org/0000-0001-9981-9910

Complete contact information is available at: https://pubs.acs.org/10.1021/acssuschemeng.0c08314

\section{Author Contributions}

The manuscript was written through contributions of all authors. All authors have given approval to the final version of the manuscript.

Notes

The authors declare no competing financial interest.

\section{ACKNOWLEDGMENTS}

The authors would like to gratefully acknowledge the Region Occitanie for the thesis grant (L.G.) and the CNRS, University Paul Sabatier, IMT Mines Albi and JSPS for financial support. The ICT facilities (ICT, Toulouse, France, http://ict.ups-tlse. $\mathrm{fr} /$ ) and especially those of NMR and Mass spectrometry are gratefully acknowledged for carrying out the corresponding analyses. We also want to thank Sylvie Del Confetto, Severine Patry, and Philippe Accart for HPLC, calorimetry, and particles sizes analyses.

\section{REFERENCES}

(1) Anastas, P. T.; Warner, J. C. Green Chemistry: Theory and Practice; Oxford University Press: Oxford/New York, 1998.

(2) Poliakoff, M.; Licence, P. Green Chemistry. Nature 2007, 450 (7171), 810-812.

(3) Varma, R. S. Chemical Activation by Mechanochemical Mixing, Microwave and Ultrasonic Irradiation. Green Chem. 2008, 10 (11), $1129-1130$

(4) Baig, R. B. N.; Varma, R. S. Alternative Energy Input: Mechanochemical, Microwave and Ultrasound-Assisted Organic Synthesis. Chem. Soc. Rev. 2012, 41 (4), 1559-1584.

(5) Stauch, T.; Dreuw, A. Advances in Quantum Mechanochemistry: Electronic Structure Methods and Force Analysis. Chem. Rev. 2016, 116 (22), 14137-14180.

(6) McNaught, A. D.; Wilkinson, A. IUPAC. Compendium of Chemical Technology, $2^{\text {nd }}$ ed; Blackwell Scientific Publications: Oxford; 1997.

(7) Takacs, L. The Historical Development of Mechanochemistry. Chem. Soc. Rev. 2013, 42 (18), 7649-7659.

(8) Toda, F.; Tanaka, K.; Iwata, S. Oxidative Coupling Reactions of Phenols with Iron(III) Chloride in the Solid State. J. Org. Chem. 1989, 54 (13), 3007-3009.

(9) Kaupp, G. Solvent-Less Organic Synthesis. Kirk-Othmer Encyclopedia of Chemical Technology; John Wiley \& Sons, Inc.: New York, 2012; pp 1-43 DOI: 10.1002/0471238961.solvkaup.a01.

(10) Achar, T. K.; Bose, A.; Mal, P. Mechanochemical Synthesis of Small Organic Molecules. Beilstein J. Org. Chem. 2017, 13 (1), 19071931.

(11) Cintas, P.; Tagliapietra, S.; Caporaso, M.; Tabasso, S.; Cravotto, G. Enabling Technologies Built on a Sonochemical Platform: Challenges and Opportunities. Ultrason. Sonochem. 2015, $25,8-16$.

(12) Baláž, P.; Achimovičová, M.; Baláž, M.; Billik, P.; CherkezovaZheleva, Z.; Criado, J. M.; Delogu, F.; Dutková, E.; Gaffet, E.; Gotor, F. J.; Kumar, R.; Mitov, I.; Rojac, T.; Senna, M.; Streletskii, A.; Wieczorek-Ciurowa, K. Hallmarks of Mechanochemistry: From 
Nanoparticles to Technology. Chem. Soc. Rev. 2013, 42 (18), 75717637.

(13) Zhang, H.; Lin, Y.; Xu, Y.; Weng, W. Mechanochemistry of Topological Complex Polymer Systems. Top. Curr. Chem. 2014, 369, 135-207.

(14) Cintas, P.; Cravotto, G.; Barge, A.; Martina, K. Interplay Between Mechanochemistry and Sonochemistry. Top. Curr. Chem. 2014, 369, 239-284.

(15) Lukin, S.; Stolar, T.; Tireli, M.; Blanco, M. V.; Babić, D.; Friščić, T.; Užarević, K.; Halasz, I. Tandem In Situ Monitoring for Quantitative Assessment of Mechanochemical Reactions Involving Structurally Unknown Phases. Chem. - Eur. J. 2017, 23 (56), 1394113949.

(16) Declerck, V.; Nun, P.; Martinez, J.; Lamaty, F. Solvent-Free Synthesis of Peptides. Angew. Chem., Int. Ed. 2009, 48 (49), 93189321.

(17) Oliveira, P. F. M.; Baron, M.; Chamayou, A.; Baltas, M.; Guidetti, B.; Haruta, N.; Tanaka, K.; Sato, T. Lowering the Activation Energy under Mechanochemical Conditions: The Case of 2,3Diphenylquinoxaline. ChemistrySelect 2016, 1 (5), 984-988.

(18) Gil-Gonzalez, E.; Rodriguez-Laguna, M. d. R.; SanchezJimenez, P. E.; Perejon, A.; Perez-Maqueda, L. A. Unveiling Mechanochemistry: Kinematic-Kinetic Approach for the Prediction of Mechanically Induced Reactions. J. Alloys Compd. 2021, 866, 158925.

(19) Colacino, E.; Carta, M.; Pia, G.; Porcheddu, A.; Ricci, P. C.; Delogu, F. Processing and Investigation Methods in Mechanochemical Kinetics. ACS Omega 2018, 3 (8), 9196-9209.

(20) Carta, M.; Colacino, E.; Delogu, F.; Porcheddu, A. Kinetics of Mechanochemical Transformations. Phys. Chem. Chem. Phys. 2020, 22 (26), 14489-14502.

(21) Carta, M.; James, S. L.; Delogu, F. Phenomenological Inferences on the Kinetics of a Mechanically Activated Knoevenagel Condensation: Understanding the "Snowball" Kinetic Effect in Ball Milling. Molecules 2019, 24 (19), 3600.

(22) Zhang, Z.; Peng, Z.-W.; Hao, M.-F.; Gao, J.-G. Mechanochemical Diels-Alder Cycloaddition Reactions for Straightforward Synthesis of Endo-Norbornene Derivatives. Synlett 2010, 2010 (19), 28952898.

(23) Andersen, J. M.; Mack, J. Decoupling the Arrhenius Equation via Mechanochemistry. Chem. Sci. 2017, 8 (8), 5447-5453.

(24) Andersen, J.; Brunemann, J.; Mack, J. Exploring Stable, SubAmbient Temperatures in Mechanochemistry via a Diverse Set of Enantioselective Reactions. React. Chem. Eng. 2019, 4 (7), 12291236.

(25) McKissic, K. S.; Caruso, J. T.; Blair, R. G.; Mack, J. Comparison of Shaking versus Baking: Further Understanding the Energetics of a Mechanochemical Reaction. Green Chem. 2014, 16 (3), 1628-1632.

(26) Stevenson, R.; De Bo, G. Controlling Reactivity by Geometry in Retro-Diels-Alder Reactions under Tension. J. Am. Chem. Soc. 2017, 139 (46), 16768-16771.

(27) Wang, Z.; Craig, S. L. Stereochemical Effects on the Mechanochemical Scission of Furan-Maleimide Diels-Alder Adducts. Chem. Commun. 2019, 55 (81), 12263-12266.

(28) Haruta, N.; de Oliveira, P. F. M.; Sato, T.; Tanaka, K.; Baron, M. Force-Induced Dissolution of Imaginary Mode in Mechanochemical Reaction: Dibenzophenazine Synthesis. J. Phys. Chem. C 2019, 123 (35), 21581-21587.

(29) Smalø, H. S.; Rybkin, V. V.; Klopper, W.; Helgaker, T.; Uggerud, E. Mechanochemistry: The Effect of Dynamics. J. Phys. Chem. A 2014, 118 (36), 7683-7694.

(30) Fulmer, D. A.; Shearouse, W. C.; Medonza, S. T.; Mack, J. Solvent -Free Sonogashira Coupling Reaction via High Speed Ball Milling. Green Chem. 2009, 11 (11), 1821-1825.

(31) Michalchuk, A. A. L.; Tumanov, I. A.; Boldyreva, E. V. Ball Size or Ball Mass - What Matters in Organic Mechanochemical Synthesis? CrystEngComm 2019, 21 (13), 2174-2179.
(32) Hutchings, B. P.; Crawford, D. E.; Gao, L.; Hu, P.; James, S. L. Feedback Kinetics in Mechanochemistry: The Importance of Cohesive States. Angew. Chem., Int. Ed. 2017, 56 (48), 15252-15256.

(33) Plasson, R.; Brandenburg, A.; Jullien, L.; Bersini, H. Autocatalyses. J. Phys. Chem. A 2011, 115 (28), 8073-8085.

(34) Veselovsky, V. V.; Gybin, A. S.; Lozanova, A. V.; Moiseenkov, A. M.; Smit, W. A.; Caple, R. Dramatic Acceleration of the Diels-Alder Reaction by Adsorption on Chromatography Adsorbents. Tetrahedron Lett. 1988, 29 (2), 175-178.

(35) Horváth, A. K. Law of Mass Action Type Chemical Mechanisms for Modeling Autocatalysis and Hypercycles: Their Role in the Evolutionary Race. ChemPhysChem 2020, 21 (15), 17031710 .

(36) Huskić, I.; Lennox, C. B.; Friščić, T. Accelerated Ageing Reactions: Towards Simpler, Solvent-Free, Low Energy Chemistry. Green Chem. 2020, 22 (18), 5881-5901.

(37) Galwey Andrew, K.; Brown Michael, E. A Theoretical Justification for the Application of the Arrhenius Equation to Kinetics of Solid State Reactions (Mainly Ionic Crystals). Proc. R. S. London A 1995, 450 (1940), 501-512.

(38) Galwey, A. K.; Brown, M. E. Application of the Arrhenius Equation to Solid State Kinetics: Can This Be Justified? Thermochim. Acta 2002, 386 (1), 91-98.

(39) Klemm, L. H.; Solomon, W. C.; Tamiz, A. P. Molecular Complexation and Kinetics for Diels-Alder Condensation of Naphthylalkenes with Tetracyanoethylene. J. Org. Chem. 1998, 63 (19), 6503-6510.

(40) Rosokha, S. V.; Korotchenko, V.; Stern, C. L.; Zaitsev, V.; Ritzert, J. T. Substituent-Induced Switch of the Role of ChargeTransfer Complexes in the Diels-Alder Reactions of o-Chloranil and Styrenes. J. Org. Chem. 2012, 77 (14), 5971-5981.

(41) Rowley, D.; Steiner, H. Kinetics of Diene Reactions at High Temperatures. Discuss. Faraday Soc. 1951, 10, 198-213.

(42) Linder, M.; Brinck, T. On the Method-Dependence of Transition State Asynchronicity in Diels-Alder Reactions. Phys. Chem. Chem. Phys. 2013, 15 (14), 5108-5114.

(43) To download: paste in your internet browser: cinet.chim. pagesperso-orange.fr (accessed March 5, 2021).

(44) Howard, J. L.; Brand, M. C.; Browne, D. L. Switching Chemoselectivity: Using Mechanochemistry to Alter Reaction Kinetics. Angew. Chem., Int. Ed. 2018, 57 (49), 16104-16108. 